\title{
Three-question set from Michigan Neuropathy Screening Instrument adds independent prognostic information on cardiovascular outcomes: analysis of ALTITUDE trial
}

\author{
Jelena P. Seferovic ${ }^{1}$ - Marc A. Pfeffer ${ }^{1} \cdot$ Brian Claggett $^{1} \cdot$ Akshay S. Desai $^{1}$. \\ Dick de Zeeuw ${ }^{2}$ - Steven M. Haffner ${ }^{3,4}$ - John J. V. McMurray ${ }^{5}$ • \\ Hans-Henrik Parving ${ }^{6,7,8}$. Scott D. Solomon ${ }^{1}$. Nish Chaturvedi ${ }^{9}$
}

Received: 9 August 2017 / Accepted: 25 September 2017 / Published online: 3 November 2017

(C) Springer-Verlag GmbH Germany 2017

\begin{abstract}
Aims/hypothesis The self-administered Michigan Neuropathy Screening Instrument (MNSI) is used to diagnose diabetic peripheral neuropathy. We examined whether the MNSI might also provide information on risk of death and cardiovascular outcomes.

Methods In this post hoc analysis of the Aliskiren Trial in Type 2 Diabetes Using Cardio-Renal Endpoints (ALTITUDE) trial, we divided 8463 participants with type 2 diabetes and chronic kidney disease (CKD) and/or cardiovascular disease (CVD) into independent training $(n=3252)$ and validation $(n=5211)$ sets. In the training set, we identified specific questions that were independently associated with a cardiovascular composite outcome (cardiovascular death, resuscitated cardiac arrest, non-fatal myocardial infarction/stroke, heart failure hospitalisation). We then evaluated the performance of these questions in the validation set.

Results In the training set, three questions ("Are your legs numb?', 'Have you ever had an open sore on your foot?' and 'Do your legs hurt when you walk?') were significantly
\end{abstract}

Electronic supplementary material The online version of this article (https://doi.org/10.1007/s00125-017-4485-y) contains peer-reviewed but unedited supplementary material, which is available to authorised users.

Marc A. Pfeffer

mpfeffer@rics.bwh.harvard.edu

1 Cardiovascular Division, Brigham and Women's Hospital, Harvard Medical School, 75 Francis Street, Boston, MA 02115, USA

2 Department of Clinical Pharmacy and Pharmacology, University Medical Center Groningen, University of Groningen, Groningen, the Netherlands

3 Present address: Shavano Park, TX, USA associated with the cardiovascular composite outcome. In the validation set, after multivariable adjustment for key covariates, one or more positive responses $(n=3079,59.1 \%)$ was associated with a higher risk of the cardiovascular composite outcome (HR 1.54 [95\% CI 1.28, 1.85], $p<0.001$ ), heart failure hospitalisation (HR 1.74 [95\% CI 1.29, 2.35], $p<0.001$ ), myocardial infarction (HR 1.81 [95\% CI 1.23, 2.69], $p=0.003$ ), stroke (HR 1.75 [95\% CI 1.20, 2.56], $p=0.003)$ and three-point major adverse cardiovascular events (MACE) (cardiovascular death, non-fatal myocardial infarction, non-fatal stroke) (HR 1.49 [95\% CI 1.20, 1.85], $p<0.001$ ) relative to no positive responses to all questions. Associations were stronger if participants answered positively to all three questions $(n=552,11 \%)$. The addition of the total number of affirmative responses to existing models significantly improved Harrell's $C$ statistic for the cardiovascular composite outcome $(0.70$ vs $0.71, p=0.010)$, continuous net reclassification improvement $(+22 \%[+10 \%,+31 \%]$, $p=0.027)$ and integrated discrimination improvement $(+0.9 \%[+0.4 \%,+2.1 \%], p=0.007)$.

4 Department of Medicine and Clinical Epidemiology, University of Texas Health Science Center, San Antonio, TX, USA

5 BHF Cardiovascular Research Centre, University of Glasgow, Glasgow, UK

6 Department of Medical Endocrinology, Rigshospitalet, University of Copenhagen, Copenhagen, Denmark

7 Faculty of Health Science, Aarhus University, Aarhus, Denmark

Steno Diabetes Center, Gentofte, Denmark

9 Institute of Cardiovascular Science, University College London, London, UK 
Conclusions/interpretation We identified three questions from the MNSI that provide additional prognostic information for individuals with type 2 diabetes and CKD and/or CVD. If externally validated, these questions may be integrated into the clinical history to augment prediction of $\mathrm{CV}$ events in high-risk individuals with type 2 diabetes.

Keywords Cardiovascular disease $\cdot$ Cardiovascular outcomes $\cdot$ Chronic kidney disease $\cdot$ Death $\cdot$ Michigan Neuropathy Screening Instrument $\cdot$ Type 2 diabetes

$\begin{array}{ll}\text { Abbreviations } \\ \text { ALTITUDE } & \begin{array}{l}\text { Aliskiren Trial in Type 2 Diabetes Using } \\ \text { Cardio-Renal Endpoints }\end{array} \\ \text { CKD } & \text { Chronic kidney disease } \\ \text { CVD } & \text { Cardiovascular disease } \\ \text { DPN } & \text { Diabetic peripheral neuropathy } \\ \text { IDI } & \text { Integrated discrimination improvement } \\ \text { MACE } & \text { Major adverse cardiovascular events } \\ \text { MNSI } & \text { Michigan Neuropathy Screening Instrument } \\ \text { NRI } & \text { Net reclassification improvement }\end{array}$

\section{Introduction}

Diabetic peripheral neuropathy (DPN) is a common complication of both type 1 and type 2 diabetes. It is a major cause of foot ulcers and subsequent amputations, and is also associated with higher rates of all-cause and cardiovascular mortality compared with individuals without DPN [1-3]. Poor glycaemic control is the strongest risk factor for the development of DPN; however, age, duration of diabetes, smoking and the presence of other comorbidities (dyslipidaemia, hypertension, diabetic retinopathy, nephropathy) are also associated with DPN [4, 5]. Previous studies have shown that DPN is present in $30-50 \%$ of diabetic individuals $[6,7]$. Therefore, screening and timely diagnosis of DPN is important for improving foot care and highlighting the risk of adverse cardiovascular outcomes.

While electromyography, electroneurography and sural nerve biopsy are the gold standard for diagnosing DPN, the Michigan Neuropathy Screening Instrument (MNSI), introduced in 1994, is considered to be an alternative diagnostic tool. The MNSI includes two separate assessments: a 15-item self-administered questionnaire about symptoms, and a lower extremity examination that includes inspection and assessment of vibratory sensation and ankle reflexes [8]. The MNSI was developed to diagnose DPN in clinical practice and has also been used in large clinical trials [9-11]. However, its value in risk stratification has not been ascertained. Therefore, we investigated whether the MNSI questionnaire offers additional information about risk of death and major cardiovascular events in high-risk individuals with type 2 diabetes and chronic kidney disease (CKD) and/or cardiovascular disease (CVD).

\section{Methods}

Participants The Aliskiren Trial in Type 2 Diabetes Using Cardio-Renal Endpoints (ALTITUDE, NCT00549757) was a double-blind, randomised, controlled trial conducted among 8561 participants with type 2 diabetes and CKD and/or CVD who were randomly assigned to receive aliskiren $(300 \mathrm{mg} /$ day) or placebo, added to an ACE inhibitor or angiotensin II receptor blocker. Participants were followed for a median of 2.6 years (interquartile range $2.0-3.2$ ). The trial design and results have been published previously $[12,13]$. The trial was approved by the ethics committee or institutional review board at each participating center and was carried out in accordance with the Declaration of Helsinki as revised in 2008. All patients provided written informed consent.

Of the 8561 randomised participants, 8463 completed the self-administered MNSI questionnaire (the questionnaire is included in the electronic supplementary material [ESM Methods]) at baseline and were included in this post hoc analysis (the remaining 98 participants did not complete the questionnaire). Based on the date of randomisation, we divided this cohort into independent training $(n=3252$, randomised 2007-2008) and validation sets $(n=5211$, randomised 2009-2011).

Study design In the training set, we identified specific questions that were independently associated with the cardiovascular composite outcome (cardiovascular death, resuscitated cardiac arrest, non-fatal myocardial infarction/stroke, heart failure hospitalisation). These questions were then evaluated in the validation set in models adjusted for the randomised study treatment (model 1) and baseline covariates (model 2) to confirm their potential independent association with clinical outcomes.

Statistical analysis Baseline characteristics were stratified by study group (training set/validation set). Continuous data are presented as the mean $\pm \mathrm{SD}$, except for the triacylglycerol and urinary albumin-tocreatinine ratio data, which are presented as median (interquartile range). Categorical variables are expressed as proportions and were compared by the $\chi^{2}$ test, while continuous variables were compared using $t$ tests or Wilcoxon rank-sum tests as appropriate. Using the training dataset, forward stepwise-selection techniques were used with threshold $p$ values at 0.05 to identify specific questions that were independently associated with the cardiovascular composite outcome without adjustment for any other variables. We further tested the null hypothesis that all selected questions 
Table 1 Baseline characteristics by study group

\begin{tabular}{|c|c|c|}
\hline Characteristic & $\begin{array}{l}\text { Training } \\
N=3252\end{array}$ & $\begin{array}{l}\text { Validation } \\
N=5211\end{array}$ \\
\hline Age (years) & $65.2 \pm 9.6$ & $64.1 \pm 9.8$ \\
\hline Female sex & $944(29.0)$ & $1760(33.8)$ \\
\hline \multicolumn{3}{|l|}{ Race } \\
\hline White & $2024(62.2)$ & $2778(53.3)$ \\
\hline Black & $96(3.0)$ & $179(3.4)$ \\
\hline Asian & $861(26.5)$ & $1810(34.7)$ \\
\hline Native American & $3(0.1)$ & $6(0.1)$ \\
\hline Pacific Islander & $8(0.2)$ & $6(0.1)$ \\
\hline Other & $260(8.0)$ & $432(8.3)$ \\
\hline Weight $(\mathrm{kg})$ & $84.7 \pm 19.5$ & $81.8 \pm 19.3$ \\
\hline BMI $\left(\mathrm{kg} / \mathrm{m}^{2}\right)$ & $30.3 \pm 5.9$ & $29.7 \pm 5.9$ \\
\hline \multicolumn{3}{|l|}{ Duration of diabetes } \\
\hline$<1$ years & $106(3.3)$ & $180(3.5)$ \\
\hline $1-5$ years & $416(12.8)$ & $793(15.2)$ \\
\hline$>5$ years & $2730(83.9)$ & $4238(81.3)$ \\
\hline \multicolumn{3}{|l|}{ Smoking history } \\
\hline Never & $1539(47.3)$ & $2709(52.0)$ \\
\hline Ex-smoker & $1270(39.1)$ & $1825(35.0)$ \\
\hline Current smoker & $443(13.6)$ & $677(13.0)$ \\
\hline Systolic blood pressure $(\mathrm{mmHg})$ & $137.4 \pm 16.1$ & $137.2 \pm 16.6$ \\
\hline Diastolic blood pressure $(\mathrm{mmHg})$ & $74.2 \pm 9.7$ & $74.2 \pm 9.9$ \\
\hline $\mathrm{HbA}_{1 \mathrm{c}}(\%)$ & $7.7 \pm 1.6$ & $7.9 \pm 1.7$ \\
\hline $\mathrm{HbA}_{1 \mathrm{c}}(\mathrm{mmol} / \mathrm{mol})$ & $61 \pm 18$ & $63 \pm 19$ \\
\hline Total cholesterol (mmol/l) & $4.4 \pm 1.1$ & $4.6 \pm 1.2$ \\
\hline LDL-cholesterol (mmol/l) & $2.5 \pm 0.9$ & $2.6 \pm 1.0$ \\
\hline HDL-cholesterol (mmol/l) & $1.2 \pm 0.3$ & $1.2 \pm 0.3$ \\
\hline Triacylglycerol (mmol/l) & $1.8(1.3-2.6)$ & $1.8(1.2-2.5)$ \\
\hline $\mathrm{eGFR}\left(\mathrm{ml} / \mathrm{min} / 1.73 \mathrm{~m}^{2}\right)$ & $54.3 \pm 21.3$ & $57.7 \pm 24.0$ \\
\hline Urinary albumin-to-creatinine ratio & $26.9(4.2-82.0)$ & $36.3(8.5-111.5)$ \\
\hline \multicolumn{3}{|l|}{ Medical history } \\
\hline Chronic heart failure & $374(11.5)$ & $489(9.4)$ \\
\hline CVD & $1535(47.2)$ & $2045(39.2)$ \\
\hline Unstable angina & $364(11.2)$ & $441(8.5)$ \\
\hline Percutaneous coronary intervention & $500(15.4)$ & 707 (13.6) \\
\hline Coronary artery bypass surgery & $489(15.0)$ & $560(10.7)$ \\
\hline Hospitalisation for myocardial infarction & $585(18.0)$ & $828(15.9)$ \\
\hline Hospitalisation for stroke & $359(11.0)$ & $477(9.2)$ \\
\hline Transient ischaemic attack & $170(5.2)$ & $174(3.3)$ \\
\hline Atrial fibrillation & $289(8.9)$ & $435(8.3)$ \\
\hline Amputation of toe/foot/leg & $129(4.0)$ & $209(4.0)$ \\
\hline Diabetic nephropathy & $2070(63.7)$ & $3575(68.6)$ \\
\hline Diabetic retinopathy & $1170(36.0)$ & $1968(37.8)$ \\
\hline \multicolumn{3}{|l|}{ Antihyperglycaemic agents } \\
\hline Sulfonylurea & $1156(35.5)$ & $1551(29.8)$ \\
\hline Metformin & $1553(47.8)$ & $2351(45.1)$ \\
\hline Insulin & $1748(53.8)$ & $3046(58.5)$ \\
\hline
\end{tabular}

Data are $n(\%)$, mean (SD) or median (interquartile range) 
were equally associated (i.e. equal HR) with the outcome of interest vs the alternative that one or more of the selected questions were differentially related to the outcome (i.e. one or more HRs different from the others). These selected questions were then tested as predictors in adjusted Cox proportional hazards models using the validation dataset. We used these questions in two models. First, we estimated the risk associated with an affirmative answer to any of the three questions compared with those with no affirmative answers. Next, we estimated the risk associated with each specific number of affirmative answers compared with a reference of zero affirmative answers. Proportional hazards regression models were used to assess the association between the questions and cardiovascular outcomes. Model 1 was adjusted for the randomised study treatment. Model 2 was adjusted for baseline covariates: age, sex, race, smoking status, systolic blood pressure, eGFR, urinary albumin-to-creatinine ratio, history of heart failure, myocardial infarction, stroke, atrial fibrillation, diabetic nephropathy, diabetic retinopathy, amputation, claudication, unstable angina, coronary revascularisation, duration of diabetes, $\mathrm{HbA}_{1 \mathrm{c}}$ and randomised treatment [14]. Harrell's C statistics (compared using a transformation of the equivalent Somers' D parameters [15]), continuous net reclassification improvement (NRI) and integrated discrimination improvement (IDI) were obtained by comparing the described multivariable Cox models with and without the inclusion of the variable identifying the number of 'yes' responses from among the chosen MNSI questions and was assessed at 3 years post-randomisation. Analyses were repeated for subgroups of participants based on CVD history as well as CKD status at randomisation. Assessment of the proportional hazards assumption was performed using Schoenfeld residuals.
Two-sided $p$ values $<0.05$ were considered to be statistically significant. Analyses were performed using Stata version 13.1 (Stata Corporation, College Station, TX, USA).

\section{Results}

The 8463 participants were divided into a training $(n=3252)$ and a validation group $(n=5211)$. Baseline characteristics in these two groups are presented in Table 1. Participants included in the training and validation groups were qualitatively similar. All pairwise correlations between MNSI questions were less than +0.40 . In the training dataset, we identified three questions which were independent predictors of the cardiovascular composite outcome: question 1, Are your legs and/or feet numb? (HR 1.25 [95\% CI 1.04, 1.50], $p=0.020$ ); question 8 , Have you ever had an open sore on your foot? (HR 1.44 [95\% CI 1.18, 1.76], $p<0.001$ ); and question 12, Do your legs hurt when you walk? (HR 1.62 [95\% CI 1.35, 1.95], $p<0.001$ ) (Table 2). The associations of the three questions with specific cardiovascular outcomes in the training dataset are shown in ESM Table 1. After adjusting for other baseline covariates, we found no evidence that the HRs associated with any of the three questions were significantly different from the others with respect to any of the outcomes.

In the validation set, 3079 participants $(59.1 \%)$ answered 'yes' to at least one of these three questions (29.7\% answered positively to one, $18.8 \%$ to two and $10.6 \%$ to three questions).

We then analysed the associations between different combinations of individual questions and cardiovascular outcomes in the validation dataset (Table 3, Fig. 1). In model 1, participants who answered 'yes' to at least one of the three questions demonstrated a higher risk of the cardiovascular composite outcome (HR 1.77 [95\% CI 1.48, 2.11], $p<0.001$ ), all-
Table 2 Association between the three questions and cardiovascular composite outcome, models 1 and 2 in participants randomised in 2007-2008 (training group $n=3252$ )

\begin{tabular}{|c|c|c|c|c|}
\hline & \multicolumn{2}{|l|}{ Model $1^{\mathrm{a}}$} & \multicolumn{2}{|l|}{ Model $2^{\mathrm{b}}$} \\
\hline & $\mathrm{HR}(95 \% \mathrm{CI})$ & $p$ value & $\operatorname{HR}(95 \% \mathrm{CI})$ & $p$ value \\
\hline & \multicolumn{2}{|c|}{524 events, $p=0.19$ (equality) } & \multicolumn{2}{|c|}{517 events, $p=0.73$ (equality) } \\
\hline Question 1 & $1.25(1.04,1.50)$ & 0.020 & $1.22(1.01,1.48)$ & 0.037 \\
\hline Question 8 & $1.44(1.18,1.76)$ & $<0.001$ & $1.37(1.12,1.68)$ & 0.003 \\
\hline Question 12 & $1.62(1.35,1.95)$ & $<0.001$ & $1.33(1.10,1.62)$ & 0.004 \\
\hline
\end{tabular}

Question 1, Are your legs and/or feet numb?; Question 8, Have you ever had an open sore on your foot?; Question 12 , Do your legs hurt when you walk?

${ }^{\text {a }}$ Model 1 adjusted for the randomised study treatment

${ }^{\mathrm{b}}$ Model 2 adjusted for age, sex, race, smoking status, systolic blood pressure, eGFR, urinary albumin-to-creatinine ratio, heart failure history, myocardial infarction, stroke, atrial fibrillation, diabetic nephropathy, diabetic retinopathy, amputation, claudication, unstable angina, coronary revascularisation, duration of diabetes, $\mathrm{HbA}_{1 \mathrm{c}}$ and randomised treatment 
Table 3 Association of the questions and outcomes, models 1 and 2 in participants who were randomised in 2009-2011 (validation group $n=5211)$

\begin{tabular}{lllll}
\hline Answer & Model 1 & & Model 2 \\
\cline { 2 - 3 } & HR $(95 \% \mathrm{CI})$ & $p$ value & & HR $(95 \% \mathrm{CI}) \quad p$ value \\
\hline
\end{tabular}

Cardiovascular composite outcome

$\begin{array}{lllll} & 595 \text { events } & & 587 \text { events } & \\ \text { Any 'yes' } & 1.77(1.48,2.11) & <0.001 & 1.54(1.28,1.85) & <0.001 \\ \text { All 'yes' } & 2.08(1.61,2.70) & <0.001 & 1.71(1.30,2.23) & <0.001\end{array}$

All-cause death

$\begin{array}{lllll} & 369 \text { events } & & 366 \text { events } & \\ \text { Any 'yes' } & 1.48(1.19,1.84) & <0.001 & 1.24(0.99,1.56) & 0.059 \\ \text { All 'yes' } & 2.06(1.51,2.81) & <0.001 & 1.60(1.16,2.22) & 0.005\end{array}$

Cardiovascular death

\begin{tabular}{llrll} 
& 222 events & \multicolumn{3}{l}{220 events } \\
Any 'yes' & $1.51(1.14,2.01)$ & 0.004 & $1.25(0.93,1.67)$ & 0.14 \\
All 'yes' & $2.14(1.44,3.18)$ & $<0.001$ & $1.63(1.07,2.48)$ & 0.022
\end{tabular}

Heart failure hospitalisation

$\begin{array}{llllr} & 235 \text { events } & & 232 \text { events } & \\ \text { Any 'yes' } & 2.00(1.50,2.68) & <0.001 & 1.74(1.29,2.35) & <0.001 \\ \text { All 'yes' } & 2.20(1.44,3.36) & <0.001 & 1.77(1.14,2.74) & 0.011\end{array}$

Myocardial infarction

$\begin{array}{lllll} & 145 \text { events } & & 143 \text { events } & \\ \text { Any 'yes' } & 2.20(1.51,3.21) & <0.001 & 1.81(1.23,2.69) & 0.003 \\ \text { All 'yes' } & 2.75(1.63,4.65) & <0.001 & 2.14(1.24,3.70) & 0.007\end{array}$
Stroke

$\begin{array}{llrll} & 152 \text { events } & & 149 \text { events } & \\ \text { Any 'yes' } & 1.94(1.36,2.78) & <0.001 & 1.75(1.20,2.56) & 0.003 \\ \text { All 'yes' } & 1.70(0.97,2.99) & 0.066 & 1.44(0.79,2.61) & 0.24\end{array}$

Three-point MACE (cardiovascular death, non-fatal myocardial infarction, non-fatal stroke)

\begin{tabular}{llllr} 
& 436 events & & 431 events & \\
Any 'yes' & $1.75(1.42,2.15)$ & $<0.001$ & $1.49(1.20,1.85)$ & $<0.001$ \\
All 'yes' & $2.10(1.56,2.84)$ & $<0.001$ & $1.69(1.23,2.31)$ & 0.001 \\
\hline
\end{tabular}

${ }^{\mathrm{a}}$ Model 1 adjusted for the randomised study treatment

${ }^{\mathrm{b}}$ Model 2 adjusted for age, sex, race, smoking status, systolic blood pressure, eGFR, urinary albumin-to-creatinine ratio, heart failure history, myocardial infarction, stroke, atrial fibrillation, diabetic nephropathy, diabetic retinopathy, amputation, claudication, unstable angina, coronary revascularisation, duration of diabetes, $\mathrm{HbA}_{1 \mathrm{c}}$ and randomised treatment For all reported HRs, no significant violations of the proportional hazards assumption were detected $(p>0.05$ for all)

cause death (HR 1.48 [95\% CI 1.19, 1.84], $p<0.001$ ), cardiovascular death (HR 1.51 [95\% CI 1.14, 2.01], $p=0.004$ ), heart failure hospitalisation (HR 2.00 [95\% CI 1.50, 2.68], $p<0.001$ ), myocardial infarction (HR 2.20 [95\% CI 1.51, 3.21], $p<0.001$ ), stroke (HR 1.94 [95\% CI 1.36, 2.78], $p<0.001)$ and three-point major adverse cardiovascular events (MACE) (cardiovascular death, non-fatal myocardial infarction, non-fatal stroke) (HR 1.75 [95\% CI 1.42, 2.15], $p<0.001)$ relative to those who answered 'no' to all of the questions. After multivariable adjustment, the excess risk associated with a positive answer to a question was reduced by 25-50\%, but remained clinically important and retained statistical significance (with the exceptions of all-cause death and cardiovascular death). These associations became even stronger if participants answered positively to multiple questions in relation to all cardiovascular outcomes, except for stroke (ESM Table 2). The addition of the total number of affirmative responses to existing models significantly improved Harrell's C statistic for the cardiovascular composite outcome $(0.70 \mathrm{vs}$ $0.71, p=0.010)$, continuous NRI $(+22 \%[+10 \%,+31 \%]$, $p=0.027)$ and IDI $(+0.9 \%[+0.4 \%,+2.1 \%], p=0.007)$. For all other outcomes, $\mathrm{C}$ statistic was improved by +0.00 to +0.02 , NRI by $+17 \%$ to $+25 \%$ and IDI by $+0.3 \%$ to $+0.8 \%$ (Table 4). All results were generally consistent across subgroups of participants defined by the presence or absence of CVD and CKD (ESM Tables 3-10).

\section{Discussion}

This study identified three out of the 15 simple questions from the MNSI that provide additional prognostic information about the risk of all-cause death, cardiovascular death and a composite cardiovascular outcome, as well as its components, in individuals with type 2 diabetes and CKD and/or CVD. While the MNSI questionnaire has previously been used to screen for DPN [9-11], this is the first time these questions have been used as predictors of risk of cardiovascular events. A positive answer to any one of the three identified questions was associated with a higher risk of adverse cardiovascular events, and the relationship was even stronger if the answers to multiple questions were positive. Furthermore, when participant characteristics and other cardiovascular risk factors were considered, a positive answer to each of these three questions provided additional prognostic information concerning the risk of the outcomes described.

The DCCT/EDIC study investigators examined the performance of each item in the MNSI questionnaire and examination in confirming the diagnosis of DPN. These investigators concluded that a reduced index of four questions performed nearly as well as the more extensive instrument [11]. Two of these four questions (question 1 'Are your legs and/or feet numb?' and question 8 'Have you ever had an open sore on your foot?') were also identified by us as important predictors of cardiovascular outcomes.

As cardiovascular risk factors and microvascular disease are associated with both DPN and CVD in people with type 2 diabetes, it is perhaps unsurprising that instruments designed to detect DPN might also predict future CVD. That the risks associated with simple questions are strong and only marginally attenuated by multivariable adjustment that accounts for 
Fig. 1 The association of answer 'yes' to (a) any of the three questions and (b) all three questions with adverse outcomes in participants randomised in 2009-2011 (validation group $n=5211$ ). A logarithmic scale has been used on the $x$-axis. CV, cardiovascular

$\begin{array}{lcc}\text { a Outcome } & \text { Events }(n) & \mathrm{HR}(95 \% \mathrm{Cl}) \\ & & \\ \text { Any yes } & & \\ \text { CV composite } & 587 & 1.54(1.28,1.85) \\ \text { All-cause death } & 366 & 1.24(0.99,1.56) \\ \text { CV death } & 220 & 1.25(0.93,1.67) \\ \text { HF hospitalisation } & 232 & 1.74(1.29,2.35) \\ \text { Myocardial infarction } & 143 & 1.81(1.23,2.69) \\ \text { Stroke } & 149 & 1.75(1.20,2.56) \\ \text { Three-point MACE } & 431 & 1.49(1.20,1.85)\end{array}$

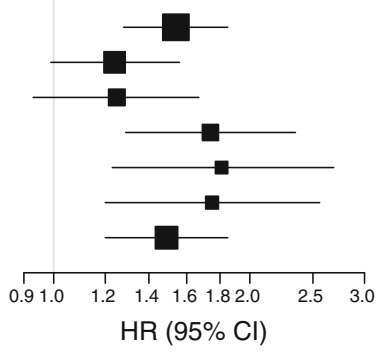

$\begin{array}{lcc}\text { b Outcome } & \text { Events }(n) & \text { HR }(95 \% \mathrm{Cl}) \\ \text { All yes } & & \\ \text { CV composite } & 587 & 1.71(1.30,2.23) \\ \text { All-cause death } & 366 & 1.60(1.16,2.22) \\ \text { CV death } & 220 & 1.63(1.07,2.48) \\ \text { HF hospitalisation } & 232 & 1.77(1.14,2.74) \\ \text { Myocardial infarction } & 143 & 2.14(1.24,3.70) \\ \text { Stroke } & 149 & 1.44(0.79,2.61) \\ \text { Three-point MACE } & 431 & 1.69(1.23,2.31)\end{array}$

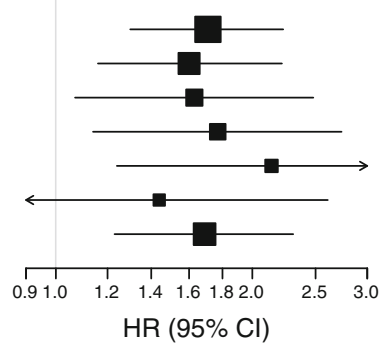

variables associated with the risk of experiencing the primary outcome of the study is more striking. The aetiological explanations for this are unclear. Alternatively, or additionally, these questions may capture unmeasured pathways (e.g. chronic inflammation in association with a history of an open sore) or more global aspects of vascular damage in association with type 2 diabetes. A similar hypothesis has been invoked to account for the repeated observation that abnormalities of retinal vascular architecture, which are thought to mimic patterns in the cerebrovascular territory, predict outcomes independent of other vascular risk factors $[16,17]$.

It is well known that cardiovascular events are a leading cause of increased morbidity and mortality rates in people with type 2 diabetes. Therefore, in clinical settings, there is a growing need for tools which could help to accurately assess the risk of adverse events in these individuals. While study of residual confounding and alternative pathways are of interest in understanding mechanisms to identify new therapeutic

Table 4 Harrell's C statistic, continuous NRI and IDI: comparison of models with and without three MNSI questions

\begin{tabular}{|c|c|c|c|c|c|c|}
\hline \multirow[t]{2}{*}{ Outcome } & \multicolumn{6}{|c|}{ With vs without ${ }^{\mathrm{a}}$ MNSI questions } \\
\hline & Harrell's C & $p$ value & NRI & $p$ value & IDI & $p$ value \\
\hline Cardiovascular composite outcome & 0.70 vs 0.71 & 0.010 & $+22 \%(+10 \%,+31 \%)$ & 0.027 & $+0.9 \%(+0.4 \%,+2.1 \%)$ & 0.007 \\
\hline All-cause death & 0.70 vs 0.71 & 0.13 & $+22 \%(-3 \%,+36 \%)$ & 0.09 & $+0.5 \%(+0.1 \%,+1.8 \%)$ & 0.020 \\
\hline Cardiovascular death & 0.72 vs 0.73 & 0.16 & $+17 \%(-5 \%,+31 \%)$ & 0.13 & $+0.3 \%(0.0 \%,+1.6 \%)$ & 0.040 \\
\hline Heart failure hospitalisation & 0.78 vs 0.79 & 0.003 & $+25 \%(+11 \%,+33 \%)$ & $<0.001$ & $+0.4 \%(-0.1 \%,+1.6 \%)$ & 0.11 \\
\hline Myocardial infarction & 0.74 vs 0.75 & 0.27 & $+24 \%(+10 \%,+34 \%)$ & 0.013 & $+0.7 \%(+0.2 \%,+2.8 \%)$ & $<0.001$ \\
\hline Stroke & 0.70 vs 0.72 & 0.049 & $+23 \%(-13 \%,+48 \%)$ & 0.09 & $+0.4 \%(-0.1 \%,+2.0 \%)$ & 0.07 \\
\hline Three-point MACE ${ }^{\mathrm{b}}$ & 0.69 vs 0.69 & 0.17 & $+22 \%(+6 \%,+36 \%)$ & 0.033 & $+0.8 \%(+0.3 \%,+2 \%)$ & 0.007 \\
\hline
\end{tabular}

${ }^{a}$ Model adjusted for age, sex, race, smoking status, systolic blood pressure, eGFR, urinary albumin-to-creatinine ratio, heart failure history, myocardial infarction, stroke, atrial fibrillation, diabetic nephropathy, diabetic retinopathy, amputation, claudication, unstable angina, coronary revascularisation, duration of diabetes, $\mathrm{HbA}_{1 \mathrm{c}}$ and randomised treatment

${ }^{\mathrm{b}} \mathrm{CV}$ death, non-fatal myocardial infarction, non-fatal stroke 
targets, current clinical practice should be more concerned with identification of high-risk individuals to whom existing therapies can be targeted. In clinical practice, complication assessment will also suffer from similar levels of imprecision as in ALTITUDE and other studies, and performance of additional tests, such as markers of inflammation, are time consuming and expensive. Inclusion of just three simple, yet strongly predictive, questions may therefore be of substantial importance in clinical practice. Prediction models are widely used in medicine as potential aids in clinical understanding and therapeutic decision-making, as well as better assessment of prognosis. However, before a new risk prediction model could be adapted in clinical practice and widely used it needs to be externally validated to assess its generalisability [18-20]. In this analysis, we demonstrated internal validation by showing that three questions identified from the derivation set provided statistically significant prognostic information in an independent validation set.

There are several limitations of this study that need to be noted. First, all participants were included in ALTITUDE based on same inclusion criteria and, although divided into statistically independent derivation and validation groups, the two groups were similar overall. Therefore, the lack of external validation, as well as the fact that these findings may not be generalisable to other populations of individuals with type 2 diabetes, is considered an important limitation. In addition, we are limited by the fact that the cohort involved in this clinical trial included only high-risk participants who were therefore more susceptible to adverse cardiovascular outcomes.

In conclusion, we believe that these three questions represent a simple, non-invasive and inexpensive tool which could potentially provide additional prediction information in clinical practice. If externally validated in people with type 2 diabetes who have a lower cardiovascular risk, these questions may be integrated into the clinical history to augment the prediction of cardiovascular events in high-risk individuals.

Acknowledgements Some of the data from this manuscript were presented as an abstract at the 53rd EASD Annual Meeting in Lisbon, 13 September 2017: Seferovic et al. Three question set from Michigan Neuropathy Screening Instrument add independent prognostic information on cardiovascular outcomes: post hoc analysis of ALTITUDE trial.

Data availability Data are available from the corresponding author on reasonable request.

Funding Novartis Pharmaceuticals was involved in the ALTITUDE trial design and protocol development, and data collection. The study sponsor was not involved in the design of this study; the collection, analysis and interpretation of data; writing the report; or the decision to submit the report for publication.
Duality of interest JPS and BC declare that there is no duality of interest associated with this manuscript.

MAP, ASD, DdZ, SMH, JJVM, HHP, SDS and NC were members of the ALTITUDE steering committee and received funding from Novartis in relation to ALTITUDE.

MAP was a consultant to Amgen, AstraZeneca, Bayer, DalCor Pharma UK, Genzyme, Lilly, Medicines Company, MedImmune, Medtronic, Merck, Novartis, Novo Nordisk, Relypsa, Salix, Sanderling, Sanofi, Takeda, Teva, Thrasos and Vericel, and has received research grant support from Amgen, Celladon, Novartis and Sanofi. The Brigham and Women's Hospital has patents for the use of inhibitors of the renin-angiotensin system in selected survivors of myocardial infarction with Novartis Pharmaceuticals. MAP is a co-inventor and his share of the licensing agreement is irrevocably transferred to charity.

ASD has received honoraria for consulting or advisory board participation from Janssen, Sanofi, Merck, St Jude Medical, AstraZeneca, and Relypsa. ASD reports grants and personal fees from Novartis during the conduct of the study; and personal fees from St Jude Medical, Relypsa, Janssen, Sanofi, Merck and AstraZeneca outside the submitted work.

DdZ's employer, University Medical Center Groningen, was paid by the following companies (trials) for time spent: AbbVie (RADAR, SONAR), Astellas, Astra Zeneca (PLANET-I, PLANET-II), Bayer, Boehringer Ingelheim, Fresenius, Janssen (CANVAS, CANVAS-R, CREDENCE), Novartis (ALTITUDE).

JJVM's employer, University of Glasgow, was paid by following companies (trials) for time spent: Cardiorentis (TRUE-AHF trial), Amgen (ATOMIC-HF and COSMIC-HF), Novartis (ATMOSPHERE, PARADIGM-HF, PARAGON-HF, PARADISE-MI), Oxford University/Bayer (ACE trial), GlaxoSmithKline (Harmony-Outcome trial, ASCEND-D and ASCEND-ND), Theracos (BEST trial), Abbvie (SONAR trial), DelCor (Dal-GenE trial), Pfizer (SPIRE trial), Merck (MK-3102 programme and for the VICTORIA trial Merck), AstraZeneca (planning a new clinical trial using dapagliflozin in heart failure), Bristol Myers Squibb planning a new clinical trial (using an HNO donor), and Kidney Research UK/Kings College Hospital, London/Vifor-Fresenius Pharma. JJNM reports other grants from Novartis, Cardiorentis, Amgen, Novartis, Oxford University/Bayer, GlaxoSmithKline, Theracos, Abbvie, DalCor, Pfizer, Merck, AstraZeneca, Bristol Myers Squibb, and Kidney Research UK/Kings College Hospital, London/Vifor-Fresenius Pharma outside the submitted work.

SDS reports research grants/consulting fees for Amgen, AstraZeneca, Sanofi, AstraZeneca, GlaxoSmithKline, and Gilead; previous consultation with Bristol Myers Squibb and Theracos; advisory board participation for Merck, and Roche; and research with Ionis.

$\mathrm{NC}$ receives payments from AstraZeneca in compensation for work as a member of a data safety and management board for a clinical trial.

Contribution statement All authors contributed to the interpretation of the results, writing or revision of the manuscript, and approved the decision to submit the article for publication. The data analysis was done by the academic authors (MAP, BC and JPS) at Brigham and Women's Hospital. MAP is the guarantor of this work.

\section{References}

1. Forsblom CM, Sane T, Groop PH et al (1998) Risk factors for mortality in type II (non-insulin-dependent) diabetes: evidence of a role for neuropathy and a protective effect of HLA-DR4. Diabetologia 41:1253-1262

2. Soedamah-Muthu SS, Chaturvedi N, Witte DR et al (2008) Relationship between risk factors and mortality in type 1 diabetic 
patients in Europe: the EURODIAB prospective complications study. Diabetes Care 31:1360-1366

3. Coppini DV, Bowtell PA, Weng C, Young PJ, Sönksen PH (2000) Showing neuropathy is related to increased mortality in diabetic patients - a survival analysis using an accelerated failure time model. J Clin Epidemiol 53:519-523

4. Ang L, Jaiswal M, Martin C, Pop-Busui R (2014) Glucose control and diabetic neuropathy: lessons from recent large clinical trials. Curr Diab Rep 1:1-15

5. Tesfaye S, Chaturvedi N, Eaton SE, et al. EURODIAB Prospective Complications Study Group (2005) Vascular risk factors and diabetic neuropathy. N Engl J Med 352:341-350

6. Tesfaye S, Boulton AJ, Dyck PJ et al (2010) Toronto Diabetic Neuropathy Expert Group. Diabetic neuropathies: update on definitions, diagnostic criteria, estimation of severity, and treatments. Diabetes Care 33:2285-2293

7. Edwards JL, Vincent AM, Cheng HT, Feldman EL (2008) Diabetic neuropathy: mechanisms to management. Pharmacol Ther 120:1-34

8. Feldman EL, Stevens MJ, Thomas PK, Brown MB, Canal N, Greene DA (1994) A practical two-step quantitative clinical and electrophysiological assessment for the diagnosis and staging of diabetic neuropathy. Diabetes Care 17:1281-1289

9. Pop-Busui R, Evans GW, Gerstein HC, et al. Action to Control Cardiovascular Risk in Diabetes Study Group (2010) Effects of cardiac autonomic dysfunction on mortality risk in the Action to Control Cardiovascular Risk in Diabetes (ACCORD) trial. Diabetes Care 33:1688-1690

10. Pop-Busui R, Lu J, Lopes N, Jones TL, BARI 2D Investigators (2009) Prevalence of diabetic peripheral neuropathy and relation to glycemic control therapies at baseline in the BARI 2D cohort. J Peripher Nerv Syst 14:1-13
11. Herman WH, Pop-Busui R, Braffett BH, et al. DCCT/EDIC Research Group (2012) Use of the Michigan Neuropathy Screening Instrument as a measure of distal symmetrical peripheral neuropathy in type 1 diabetes: results from the Diabetes Control and Complications Trial/Epidemiology of Diabetes Interventions and Complications. Diabet Med 29:937-944

12. Parving HH, Brenner BM, McMurray JJ et al (2009) Aliskiren trial in type 2 diabetes using cardio-renal endpoints (ALTITUDE): rationale and study design. Nephrol Dial Transplant 24:1663-1671

13. Parving HH, Brenner BM, McMurray JJ, et al. ALTITUDE Investigators (2012) Cardiorenal end points in a trial of aliskiren for type 2 diabetes. N Engl J Med 367: 2204-2213

14. Jhund PS, McMurray JJ, Chaturvedi N et al (2015) Mortality following a cardiovascular or renal event in patients with type 2 diabetes in the ALTITUDE trial. Eur Heart J 36:2463-2469

15. Newson RB (2010) Comparing the predictive powers of survival models using Harrell's C or Somers' D. Stata J 10:339

16. Witt N, Wong TY, Hughes AD et al (2006) Abnormalities of retinal microvascular structure and risk of mortality from ischemic heart disease and stroke. Hypertension 47:975-981

17. Cheung CY, Ikram MK, Klein R, Wong TY (2015) The clinical implications of recent studies on the structure and function of the retinal microvasculature in diabetes. Diabetologia 58:871-885

18. Altman DG, Royston P (2000) What do we mean by validating a prognostic model? Stat Med 19:453-473

19. Moons KG, Kengne AP, Grobbee DE et al (2012) Risk prediction models: II. External validation, model updating, and impact assessment. Heart 98:691-698

20. Altman DG, Vergouwe Y, Royston P, Moons KG (2009) Prognosis and prognostic research: validating a prognostic model. BMJ 338:b605 